DOI 10.1007/s10600-017-2009-0

Chemistry of Natural Compounds, Vol. 53, No. 2, March, 2017

\section{INDEX OF NATURAL OBJECTS}

Abrus precatorius L., No. 2, 257

Acanthopanax senticosus, No. 2, 273

Aconitum soongaricum var. pubescens, No. 2, 403

Aethionema diastrophis, No. 2, 379

Agaricus bisporus, No. 2, 383

Artemisia halophila Krasch., No. 2, 284

Artemisia rupestris L., No. 2, 371

Arthrinium arundinis ZSDS1-F3 from Phakellia fusca Thiele,

No. 2, 373

Aspergillus versicolor SCSIO 05772, No. 2, 354

Astragalus turczaninowii Kar. \& Kir., No. 2, 398

Astropyga radiata (Leske, 1778), No. 2, 356

Axinella sp., No. 2, 325

Carthamus tinctorius, No. 2, 318

Chroogomphus rutilus, No. 2, 383

Cinnamomum cassia (L.) J. Presl, No. 2, 234

Clathrus ruber, No. 2, 383

Climacoptera obtusifolia, No. 2, 375

Convallaria keiskei Miq., No. 2, 377

Cremastra appendiculata (D. Don) Makino, No. 2, 211

Engyodontium album, No. 2, 224

Eucommia ulmoides, No. 2, 254

Euphorbia yinshanica S.Q. Zhou \& G.H. Liu, No. 2, 295

Fomes fomentarius (L.) Fr., No. 2, 351

Formosa algae PPF from Patinopecten yessoensis, No. 2, 345

Formosa algae KMM 3553T from Patinopecten yessoensis, No. 2, 345

Fucus evanescens, No. 2, 345

Funalia trogii (Berk.) Bondartsev \& Singer, No. 2, 351

Ganoderma adspersum (Schulzer) Donk, No. 2, 351

Ganoderma adspersum, No. 2, 383

Ganoderma applanatum (Pers.) Pat., No. 2, 351

Ganoderma lucidum (Curtis) P. Karst, No. 2, 351

Guarea convergens T. D. Penn., No. 2, 312

Gyromitra esculenta (Pers.) Fr., No. 2, 351
Homalium stenophyllum Merr. et Chun, No. 2, 362

Houttuynia cordata Thunb., No. 2, 365

Incarvillea arguta, No. 2, 396

Kochia prostrata, No. 2, 375

Lactarius deliciosus, No. 2, 383

Lasianthus hartii, No. 2, 390

Lyophyllum decastes (Fr.) Singer, No. 2, 351

Macaranga rubiginosa, No. 2, 215

Melanoleuca cognata, No. 2, 383

Microcos tomentosa Sm. (= Grewia paniculata L.), No. 2, 394

Nocardiopsis alba from Anthogorgia caerulea, No. 2, 338

Oxytropis ochrocephala Bunge, No. 2, 322

Penicillium thomii KMM 4667 from Zostera marina, No. 2, 290

Phlomoides tuberosa Moench (= Phlomis tuberosa L.), No. 2,269

Phoma sp. (collection No. L28) from Myoporum bontioides A. Gray, No. 2, 237

Pleurotus ostreatus (Jacq.) P. Kumm., No. 2, 351

Pleurotus ostreatus, No. 2, 383

Polygonum cuspidatum, No. 2, 368

Primula veris L. var. columnae (Ten.) B. Bock., No. 2, 386

Pulicaria uliginosa, No. 2, 299

Rhizopogon luteolus, No. 2, 383

Rhodosporidium paludigenum PT3, No. 2, 406

Robinia pseudoacacia L., No. 2, 359

Russula delica Fr., No. 2, 351

Sargassum muticum, No. 2, 219

Stachys chinensis Bunge ex Benth., No. 2, 388

Streptomyces sp. from Sedum sp., No. 2, 400

Teucrium orientale L. subsp. glabrescens (Hausskn. ex

Bornm.) Rech.f, No. 2, 381

Undaria pinnatifida No. 2, 345

Viburnum erosum, No. 2, 265

Xylaria sp. C-2, No. 2, 227 